\title{
ESCALA DE ESTILOS DE APRENDIZAGEM EM SITUAÇÕES DE USO DE TECNOLOGIAS: BUSCA POR EVIDÊNCIAS DE VALIDADE DE CONTEÚDO
}

\author{
Rodrigo Hipólito Roza \\ Pontifícia Universidade Católica de Campinas - PUC-Campinas \\ Solange Muglia Wechsler \\ Pontifícia Universidade Católica de Campinas - PUC-Campinas \\ Tatiana de Cassia Nakano \\ Pontifícia Universidade Católica de Campinas - PUC-Campinas
}

\begin{abstract}
Resumo
Esse estudo teve como objetivo a busca por evidências de validade de conteúdo de uma nova escala, em desenvolvimento, intitulada Escala de Estilos de Aprendizagem em Situações de Uso de Tecnologias. Para tanto, quatro juízes na área de avaliação psicológica avaliaram os 40 itens que compõem a escala, considerando quatro estilos de aprendizagem: ativo, reflexivo, teórico e pragmático, estabelecidos por Alonso, Gallego e Honey (1997). Originalmente, a maioria dos itens $(n=34)$ apresentou índices de concordância acima de $75 \%$, mostrando-se satisfatórios. Duas novas rodadas de avaliação foram conduzidas com os itens que foram reformulados ou substituídos por novos, sendo que, após esse procedimento, atingiram o índice de concordância esperado. As análises dos coeficientes Kappa evidenciaram índices acima de 0,75. Os resultados apontaram a adequação da escala aos conteúdos do modelo que pretende avaliar, mostrando-se adequada para uso em outros estudos, empíricos, de investigação de suas qualidades psicométricas.
\end{abstract}

Palavras-chave: Estilo de aprendizagem; Tecnologia; Tecnologia da informação; Avaliação psicológica; Validade do teste.

\section{LEARNING STYLES SCALE IN TECHNOLOGY USE SITUATIONS: SEARCH FOR CONTENT VALIDITY EVIDENCES}

\begin{abstract}
This study aimed to search for evidence of content validity of the Learning Styles Scale in Technology use situations, a scale in development. For that, four judges working in the psychological assessment evaluated the 40 items of the scale, considering four learning styles: active, reflective, theoretical, and pragmatic, proposed by Alonso, Gallego and Honey (1997). Originally, most of the items $(n=34)$ showed concordance rates above $75 \%$, being satisfactory. Two new rounds of evaluation were conducted with the items that have been rewritten or replaced by new ones, and after this procedure reached the expected level of agreement. The analyzes showed Kappa coefficients indices above 0.75 . The results showed the adequacy of the scale to the contents of the model that it intends to evaluate, being suitable for use in other studies involving empirical research of its psychometric qualities.
\end{abstract}

Keywords: Learning style; Technology; Information technology; Psychological assessment; Test validity. 


\title{
ESCALA DE ESTILOS DE APRENDIZAJE EN SITUACIONES DE USO DE TECNOLOGÍAS: BÚSQUEDA DE EVIDENCIAS DE VALIDEZ DE CONTENIDO
}

\begin{abstract}
Resumen
Este estudio tuvo como objetivo la búsqueda de evidencias de validez de contenido de la Escala de Estilos de Aprendizaje en Situaciones de Uso de Tecnologías, una escala en el proceso de desarrollo. Por lo tanto, cuatro jueces en el área psicológica evaluaron los 40 ítems de la escala, considerando cuatro estilos de aprendizaje: activo, reflexivo, teórico y pragmático, propuesto por Alonso, Gallego y Honey (1997). Originalmente, la mayoría de los artículos $(n=34)$ mostraron tasas de concordancia por encima de $75 \%$, siendo satisfactoria. Se realizaron dos nuevas rondas de evaluación con los elementos que se han vuelto a trabajar o reemplazados por otros nuevos, $y$, después de este procedimiento, alcanzado el nivel esperado de acuerdo. Los análisis mostraron coeficientes Kappa índices por encima de 0,75. Los resultados mostraron la adecuación de la escala al contenido del modelo que tiene la intención de evaluar, siendo adecuada para su uso en otros estudios de investigación empírica de sus cualidades psicométricas.
\end{abstract}

Palabras clave: Estilo de aprendizaje; Tecnología; Tecnología de la información; Evaluación psicológica; Validación de test.

\section{INTRODUÇÃO}

Historicamente, os estilos individuais têm sido investigados sob diferentes denominações em função do interesse dos pesquisadores pelas variações individuais nos modos de pensamento, tais como: estilos cognitivos, intelectuais, de pensamento, de aprendizagem, de ensino, dentre outros. Tomado como uma categoria mais ampla, os estilos intelectuais, também chamados de estilos cognitivos, abarcam todas as demais categorias de estilo, mostrando-se o mais completo teoricamente (Oliveira, Inácio, \& Buriolla, 2016). De acordo com os mesmos autores, os estilos intelectuais não se referem ao nível de inteligência do individuo, mas à forma como ele emprega as suas habilidades intelectuais no dia a dia, para processar informações, obter novos conhecimentos e lidar com problemas.

Dentro dessa categoria maior intitulada estilos, os estilos de aprendizagem têm sido amplamente estudados por diversos pesquisadores ao longo das últimas quatro décadas, dada sua relevância na compreensão das preferências dos indivíduos ao aprender, a possibilidade de seu uso na adaptação das estratégias de ensino a determinados conteúdos, bem como no desenvolvimento de processos mais eficientes de aprendizagem. Associado originalmente como domínio teórico da Psicologia, o interesse na sua investigação espalhou-se para outras áreas, notadamente Educação, passando a influenciar o processo de ensino e aprendizagem. Por esse motivo, têm sido um tema amplamente discutido em contextos acadêmicos, pedagógicos e comerciais ( $\mathrm{Li}$, Medwell, Wray, \& Liu, 2016).

Os estilos apresentam como foco de interesse não o conteúdo ou eficácia com que a pessoa aprende, mas a forma como ela se comporta durante o aprendizado (Butzke \& Alberton, 2017), referindo-se às qualidades e preferências apresentadas por um indivíduo durante o processo de ensino-aprendizagem. 
Composto por uma série de características que auxiliam os alunos a perceber, interagir e responder a um ambiente de aprendizagem, os estilos de aprendizagem têm sido definidos como uma síntese de elementos ambientais, emocionais, sociológicos, físicos e psicológicos que permitem, aos indivíduos, receber, armazenar e usar seus conhecimentos e habilidades no processo de ensino e aprendizagem (Gungor, Sofraci, Çelik, \& Yayli, 2016). Envolvem o conhecimento da forma como os estudantes aprendem, como devem ser ensinados e como interagem com o ambiente de aprendizagem, fazendo-se notar diferenças entre eles em termos de processamento, sentido e utilização de informação em novas situações (Ozdemir, Alaybeyoglu, Mulayin, \& Balbal, 2016). Por esses motivos, tal conceito tem influenciado a compreensão dos professores acerca das diferenças individuais, atuado diretamente na motivação dos alunos, bem como na promoção do ambiente em que o ensino ocorre, possibilitando um maior cuidado na elaboração dos planos de aula e na avaliação individualizada (Li et al., 2016).

A forma de estudar pode fazer diferença, tanto em relação a benefícios de curto prazo quanto a longo prazo, na educação. Isso porque a conscientização dos alunos acerca de seus estilos de aprendizagem e a utilização dessa informação no ambiente em que se aprende têm apresentado resultados significativos (Gocus \& Ertek, 2016), permitindo a definição de um contexto mais apropriado, assim como o estabelecimento de um processo de intervenção, quando necessário, voltado à potencialização da aprendizagem (Kolb, 1984). Nesse sentido, a utilização dos recursos de tecnologias de informação e comunicação nas perspectivas de aprendizagem tem se mostrado abundante em termos de vantagens, ainda que algumas limitações tenham sido reconhecidas, tais como a falta de supervisão e assistência do professor (Rajper, Shaikh, Shaikh, \& Mallah, 2016). Mudanças têm sido notadas tanto na forma de aprender como na forma de ensinar, de maneira que tal temática deve ser cada vez mais investigada pelas diferentes ciências interessadas no processo de ensinoaprendizagem.

Dada sua relevância, uma série de teorias psicológicas foram desenvolvidas tendo como foco, direta ou indiretamente, os estilos de aprendizagem, na tentativa de descrever as diferenças observadas entre as preferências dos alunos para a aprendizagem. Dentre elas, pode-se citar a teoria da aprendizagem experiencial de Kolb (1984), estilos de aprendizagem de Dunn e Dunn (1978), a teoria de autogoverno mental de Sternberg (1988), estilos de aprendizagem e de ensino de Gregorc (1982) ou ainda o modelo de estilos de aprendizagem de Felder e Silverman (1988), dentre outros.

Como consequência, diversos instrumentos para avaliar estilos de aprendizagem foram construídos. Dentre estes, os inventários mais frequentemente utilizados, segundo a literatura científica (Gocus \& Ertek, 2016), são: o Kolb Learning Style Inventory (LSI), o Learning Style Inventory and 
Productivity Environmental Preference Survey Learning, Learning Styles Questionnaire (LSQ), o Index of Learning Styles (ILS), Cuestionario HoneyAlonso de Estilos de Aprendizaje (CHAEA), Survey of Study Habits and Attitudes, Learning and Study Skills Inventory, Inventory of Learning Processes e Study Process Questionnaire.

Convém destacar, no entanto, que a amplitude encontrada em relação à diversidade de estilos de aprendizagem deve ser compreendida perante o fato de que muitos deles se basearam em teorias e modelos distintos, resultando em uma grande variedade de instrumentos com classificações heterogêneas. Isso porque, ainda que nas últimas décadas, tal conceito tenha se tornado popular no campo da educação, sua terminologia ampla, assim como suas diferentes possibilidades de categorização e conceituação acabou por produzir problemas relacionados a uma definição consensual e até mesmo em relação às evidências de validade, precisão e baixa ou inexistente capacidade preditiva, apresentadas pela maior parte dos instrumentos ( $\mathrm{Li}$ et al., 2016). No entanto, apesar dessas preocupações, a expansão de interesse pelos estilos de aprendizagem tem sido intensa, notadamente em programas de desenvolvimento profissional Infelizmente tal interesse não tem sido acompanhado, segundo Cuevas (2015), pelos leigos no mundo dos negócios; administradores e professores não estão familiarizados com as questões relacionadas às evidências psicométricas dos instrumentos de medida, de modo que uma incongruência se faz presente: de um lado a prosperidade da área e, de outro, a falta de suporte empírico.

Junte-se, a esse fato, a observação de que a maioria dos instrumentos de avaliação de estilos de aprendizagem encontra-se publicada na língua inglesa, sendo utilizada por universidades e empresas em países como Estados Unidos, Grã-Bretanha e Canadá (Cué, Rincón, \& García, 2009). Especificamente no Brasil, uma lacuna se faz presente, não havendo, até o momento, nenhum instrumento que se pauta nessa concepção (Oliveira, Santos, \& Scacchetti, 2016), aprovado para uso profissional pelo Conselho Federal de Psicologia. O que se encontram são propostas de instrumentos que passaram por estudos de investigação de suas qualidades psicométricas, mas cujas pesquisas de todas essas qualidades não foram completados ou não chegaram a ser disponibilizados. Podem ser citados, como exemplos, esforços de alguns pesquisadores no processo de adaptação e/ou busca por evidências de validade de instrumentais voltados aos estilos de aprendizagem (Barros, 2009; Portilho, 2004). Assim, é importante destacar a necessidade de construir ou adaptar instrumentos de avaliação de estilos de aprendizagem que sejam voltados para a realidade brasileira.

Além disso, nota-se também que, ao longo das últimas décadas, importantes teorias e modelos de estilos de aprendizagem foram concebidos e muitos dos instrumentos de avaliação foram construídos. A sociedade passou por profundas transformações sociais, tecnológicas, econômicas e culturais que, 
conjuntamente, resultaram em uma nova forma de sociedade (Castells, 2010). Dada a relevância de tais transformações, várias denominações foram empregadas para rotulá-la, como sociedade da informação ou sociedade do conhecimento, sustentada nas tecnologias da informação e comunicação (Coll \& Monereo, 2010).

Diante dessa realidade, os estilos de aprendizagem passaram a ser valorizados como ferramentas capazes de auxiliar no processo de melhoria do uso de metodologias ativas, de forma a contribuir para a evolução do uso de tecnologias de informação no ensino (Butzke \& Alberton, 2017). Diferentes estudos foram conduzidos nesse foco, voltados à aplicação dos estilos de aprendizagem em contextos de uso de tecnologias, podendo-se citar, como exemplo, o desenvolvido por Okada, Barros e Santos (2008), no qual os autores discutiram os estilos de aprendizagem com tecnologias no âmbito de um projeto de aprendizagem aberta. Já Alves, Miranda e Moraes (2014) estudaram o uso de recursos digitais em um ambiente colaborativo, com base nos estilos de aprendizagem identificados através do questionário CHAEA, o qual já foi, inclusive, traduzido e adaptado para a realidade universitária brasileira (Portilho, 2004).

Embora exista uma variedade grande de instrumentos de avaliação de estilos de aprendizagem, principalmente no âmbito internacional, os instrumentos voltados especificamente para situações de uso das tecnologias são escassos. Dentre os 38 instrumentos de avaliação de estilos de aprendizagem identificados por Cué et al. (2009), apenas o questionário de Barros (2009) se destaca como sendo especificamente voltado para um contexto de uso de tecnologias. Tal fato contrasta com a importância das tecnologias da informação e comunicação na atual sociedade.

Ao considerar as situações de uso das tecnologias da informação e comunicação, não se deve enfatizar a tecnologia em si, mas sim o contexto mais amplo em que os indivíduos estão inseridos. Uma vez que os estilos não são fixos e podem mudar para atender a diferentes situações (Sternberg, 1994), a avaliação dos indivíduos em situações de uso de tecnologias busca ampliar a compreensão sobre suas preferências de aprender na nova realidade que os cerca. Não se pode deixar de citar que o uso dessas tecnologias, visualizadas como uma oportunidade para melhorar a qualidade de vida das pessoas nos mais diferentes ambientes de aprendizagem, tem se mostrado fator chave para a sociedade atual (Acosta-Castillo, 2016), juntamente com a identificação das capacidades intelectuais e dos recursos tecnológicos necessários para a obtenção da informação e formação.

De acordo com Ozdemir et al. (2016), no contexto da atual sociedade, as informações podem ser transferidas aos alunos em um ambiente de aprendizagem tradicional ou em ambientes de aprendizagem online. No primeiro caso, tem se mostrado um desafio para os professores satisfazer as diferentes 
exigências de aprendizagem dos alunos, dado o grande número de estudantes em cada sala de aula. Uma maneira de superar esses desafios seria, conforme o autor, a utilização de ambientes de aprendizagem online, considerando os estilos preferenciais de aprender dos alunos. Nesse sentido, o segundo caso, ambiente online, tem apresentado uma série de vantagens, notadamente ao permitir a utilização de recursos tecnológicos e multimídias (auditiva, textual, visual ou uma combinação delas, através das facilidades oferecidas pelas tecnologias de informação e comunicação).

A partir das lacunas apontadas anteriormente, assim como a relevância dos estilos de aprendizagem e a necessidade de que este conceito acompanhe as mudanças atuais da sociedade da informação, a Escala de Estilos de Aprendizagem em Situações de Uso de Tecnologias, objeto de estudo da presente pesquisa, foi desenvolvida. A escala foi baseada principalmente nos estudos de Alonso, Gallego e Honey (1997), os quais definem os estilos de aprendizagem como traços cognitivos (tais como dependência-independência de campo, contextualização e categorização, relatividade frente a impulsividade, modalidades sensoriais), afetivos (desejo e disposição atitudinal para aprender, motivação e expectativa) e fisiológicos (envolvendo diferentes biotipos e biorritmos) que servem como indicadores relativamente estáveis de como os alunos percebem, interagem e respondem a seus ambientes de aprendizagem.

$O$ instrumento aqui relatado apresenta-se como uma proposta de avaliação de quatro estilos de aprendizagem: ativo, reflexivo, teórico e pragmático, conforme definições encontradas em Alonso et al. (1997) e Mayaute (2011). O estilo ativo inclui indivíduos que se envolvem com experiências novas, gostam de desafios e trabalhos com outras pessoas. O estilo reflexivo inclui pessoas observadoras e que analisam suas experiências sob diferentes perspectivas antes de chegar a uma conclusão. Já o estilo teórico abrange pessoas que adaptam e integram suas observações em teorias mais completas e bem fundamentadas, que analisam logicamente e racionalmente a informação. $O$ estilo pragmático, por fim, inclui pessoas que experimentam ideias, teorias e técnicas novas, buscando comprovar como elas funcionam na prática.

Para que um instrumento possa ser disponibilizado para uso comercial, suas qualidades psicométricas devem ser investigadas por meio de uma série de estudos. Neste sentido, a presente pesquisa constitui-se em um primeiro estudo com a escala desenvolvida. Assim, esta pesquisa teve como objetivo a busca pelas suas evidências de validade de conteúdo da Escala de Estilos de Aprendizagem em Situações de Uso de Tecnologias. Esse tipo específico de evidência de validade busca investigar a clareza, representatividade e relevância dos itens por meio da análise de juízes, sendo recomendado antes da aplicação empírica (Alexandre \& Coluci, 2011). 


\section{MÉTODO}

\section{Participantes}

Quatro colaboradores atuaram como juízes, sendo dois doutores e dois doutorandos em psicologia. Os doutores estavam com pós-doutorado em andamento. Todos os juízes possuíam titulação de mestre na área de psicologia e estavam desenvolvendo seus trabalhos dentro da linha de pesquisa "instrumentos e processo de avaliação psicológica". Tal fato garantiu que os juízes possuíssem conhecimentos na área de avaliação psicológica. Um deles, em particular, trabalhava com a temática de estilos.

\section{Instrumentos}

Escala de Estilos de Aprendizagem em Situações de Uso de Tecnologias, construída pelo primeiro autor desse estudo. Trata-se de um instrumento informatizado para avaliar os estilos de aprendizagem de adultos em situações em que as Tecnologias da Informação e Comunicação (TICs) são empregadas.

O instrumento continha uma página inicial na qual deveriam ser preenchidas informações sociodemográficas sobre o respondente (tais como iniciais, sexo, idade, nível de escolaridade). Posteriormente, a escala em si foi apresentada, em formato eletrônico contendo as instruções e, seguidamente, seus itens. $O$ instrumento é composto por 40 itens, para os quais o participante deve responder marcando uma das alternativas, "sim" ou "não". Conforme dito anteriormente, os itens foram criados para abrangerem quatro estilos de aprendizagem: ativo, reflexivo, teórico e pragmático, baseado, em especial, no modelo teórico desenvolvido por Alonso et al. (1997). As definições de cada estilo são apresentadas posteriormente, na seção de resultados.

\section{Procedimentos}

A escala foi construída fazendo uso de termos comuns para usuários das tecnologias da informação e comunicação, tais como computador, tablet, smartphone, aplicativos (programas de computador), conteúdos digitais (apresentações, texto, som, imagem em formato digital), Internet, sites, páginas (da web), e-mail, mensagem eletrônica, grupo de discussão, redes sociais.

O projeto foi submetido à avaliação do Comitê de Ética em Pesquisa da PUC-Campinas, tendo sua execução aprovada (CAAE: 56450716.3.0000.5481). Os juízes foram convidados a participar da pesquisa e assinaram um Termo de Consentimento Livre e Esclarecido (TCLE), confirmando sua participação.

Um formulário de avaliação foi elaborado e encaminhado aos juízes contendo dois diferentes campos. O primeiro contemplava informações de identificação dos juízes, tais como identificação de nome, titulação e data. 0 segundo campo apresentava informações sobre a pesquisa e seu objetivo, definição conceitual do construto avaliado e as dimensões envolvidas, bem como 
instruções para avaliação da escala (esclarecendo que os juízes deveriam avaliar cada item da escala com base na definição de cada estilo de aprendizagem apresentada). Para isso, uma taxonomia de cada estilo foi desenvolvida pelos pesquisadores a fim de guiar o processo de classificação a ser realizado pelos juízes, sendo tal definição oferecida no formulário a eles entregue: Ativo (preferem atividades novas e desafiadoras, bem como aprendem melhor através de experiência e trabalho com outras pessoas), Reflexivo (preferem ver as coisas a partir de diferentes perspectivas, com uma oportunidade de planejar de forma antecipada, prestar atenção em detalhes e examinar significados), Teórico (conseguem aprender melhor através de modelos, teorias ou conceitos, bem como analisando ideias de forma lógica em ambientes estruturados) e Prático (possuem mais facilidade de aprender através de aplicações práticas e imediatas de atividades, com uma relação clara entre o assunto tratado e aplicações da vida real).

Aos participantes, era solicitado que indicassem o estilo de aprendizagem contemplado em cada item, segundo sua avaliação. Dessa forma, na coluna correspondente do formulário de avaliação, os juízes deveriam marcar " $A$ " para ativo, " $\mathrm{R}$ " para reflexivo, " $\mathrm{T}$ " para teórico e " $\mathrm{P}$ " para pragmático após análise de cada frase. Importante salientar que os itens foram dispostos de modo aleatório, a fim de que não pudessem ser, facilmente, identificados dada a ordem ou proximidade no momento de apresentação, evitando-se ainda que estivessem agrupados de acordo com os estilos que representavam. Após o recebimento dos formulários com os resultados da avaliação, uma tabela única foi elaborada pelos autores, contendo os estilos de aprendizagem apontados pelos juízes para cada item da escala.

Com o propósito de identificar a adequação dos itens aos estilos de aprendizagem, foi feita a análise dos índices de concordância entre os juízes. Um dos pontos controversos na análise dos índices de concordância é o número e a qualificação dos juízes. Lynn (1986 citado por Alexandre \& Coluci, 2011) recomenda de cinco a dez juízes. De acordo com os autores, na decisão acerca do número de juízes, deve-se levar em conta, dentre outros fatores, a formação, qualificação e disponibilidade dos profissionais. Assim, os critérios de inclusão selecionados para o estudo envolveram a necessidade de formação na área de avaliação psicológica, encontrar-se matriculado em curso de pós-graduação nível doutorado ou já possuir esse nível educacional, bem como apresentar conhecimentos na temática de estilos e apresentar conhecimento metodológico sobre construção de instrumentos. Desse modo, o número de possíveis participantes ficou bastante limitado. Consequentemente, ainda que a literatura recomende um número maior de juízes, este estudo, em particular, acabou por envolver um número menor de juízes (quatro ao invés do cinco recomendados), privilegiando suas qualificações. 


\section{Plano de análise dos dados}

Um banco de dados foi criado contendo as respostas dadas por cada juiz, para cada item, bem como uma coluna intitulada "juiz ideal", que correspondia ao estilo para a qual o item foi idealizado. Com auxílio do programa estatístico Statistical Package for Social Sicences - SPSS 18.0, as análises foram conduzidas. Para a condução da análise dos dados, dois métodos foram selecionados. O primeiro deles, estimativa do índice de concordância (IC) entre os juízes independentes. Para isso, as respostas dos juízes foram contabilizadas em relação a qual estilo ela representaria, de acordo com o julgamento de cada um. A porcentagem de concordância foi estimada para cada item. Considerandose que a literatura tem recomendado, usualmente, valores em torno de $80 \%$ de concordância entre os juízes como indicativo da pertinência do item (Pasquali, 2010) e que, neste estudo quatro colaboradores participaram como juízes, este valor foi aproximado para 75\%, exigindo-se que, neste caso, 3 dos 4 avaliadores concordassem em relação à categoria a qual pertenceria o item.

O segundo método consistiu na estimativa do coeficiente Kappa para cada juiz, com o objetivo de verificar a concordância interobservador, ou seja, a intensidade da concordância entre juízes. O Kappa mede o grau de concordância das avaliações nominais ou ordinais, feitas por vários avaliadores, sendo uma medida baseada no número de respostas concordantes e um dos índices mais utilizados para esse objetivo (Fonseca, Silva, \& Silva, 2007). É calculado em razão da proporção de vezes que os juízes concordam (corrigido por concordância devido ao acaso), com a proporção máxima de vezes que os juízes poderiam concordar (Alexandre \& Coluci, 2011).

O coeficiente Kappa permite estimar a significância estatística com que juízes especialistas concordam e também o seu intervalo de confiança, de modo a indicar o quanto legítimas são as interpretações. Assim, a hipótese nula, testada neste estudo, afirmava que não há concordância entre avaliadores no julgamento do estilo de aprendizagem que cada item pertence. Para isso, além da estimativa da porcentagem de concordância, determinou-se também o nível de significância (valor p) em 0,05 (95\%) como critério para que a hipótese nula fosse rejeitada.

Uma vez que o valor do coeficiente varia entre zero (nenhuma concordância) e 1 (concordância total), considerou-se que valores de Kappa acima de 0,75 indicariam uma concordância excelente; entre 0,40 e 0,75, uma concordância satisfatória; e abaixo de 0,40, uma concordância insatisfatória, recomendados por Fleiss (1981). Para tanto, cada classificação efetuada pelos juízes foi comparada com um "juiz ideal", o qual representava a classificação originalmente pensada para cada item.

A análise das respostas fornecidas por cada juiz foi realizada considerando-se o número de itens que ele classificou em cada um dos quatro estilos, número de acertos (das classificações feitas, quantas estavam de acordo 
com a classificação tomada como "correta" pelos pesquisadores) e porcentagem de acerto (considerando-se o número de classificações corretamente realizadas). O coeficiente Kappa foi estimado para cada juiz, representando a intensidade de sua concordância com os demais juízes, bem como o valor do nível de significância (valor de $p$ ) para aceitar ou rejeitar a hipótese nula.

\section{RESULTADOS}

Para análise das respostas dos juízes, os itens foram agrupados de acordo com o estilo de aprendizagem a que se referiam. A Tabela 1 apresenta os índices de concordância para os itens dos estilos ativo e reflexivo.

O estilo ativo refere-se à preferência do indivíduo por atividades novas e desafiadoras, bem como à valorização da experiência e do trabalho coletivo. Na análise dos seus itens, é possível observar que todos os itens alcançaram resultados satisfatórios, indicando a pertinência dos itens ao estilo que se propõem a avaliar. Três dos dez itens obtiveram índices de concordância de $100 \%$, sendo que, os outros sete, apresentaram índices de concordância de $75 \%$.

Importante salientar que seis itens do estilo ativo tiveram classificações divergentes provenientes da análise de um mesmo juiz, o qual, na maioria dos casos, optou por categorizar os itens no estilo teórico. Tal fato sugere uma compreensão distinta daquela esperada para o estilo por parte desse juiz, a qual se propagou para a avaliação da maior parte dos itens que compõem esse estilo. Já os demais juízes convergiram para a classificação esperada, conforme o estilo para o qual, teoricamente, o item foi desenvolvido, considerando-se também a descrição (taxonomia) fornecida no formulário por eles recebido.

O segundo estilo avaliado, o reflexivo, foi definido como aquele cujos indivíduos possuem preferência por observar as coisas a partir de diferentes pontos de vista e, dessa forma, planejar e se concentrar em detalhes e significados. Para esse estilo, dois itens apresentaram índices de concordância de $100 \%$, cinco itens apresentaram 75\% e três itens apresentaram 50\%. Desse modo, sete dos 10 itens apresentaram concordância esperada, mostrando-se adequados para o conteúdo que pretendem representar. Destaque deve ser dado ao item 6 que, embora tenha apresentado concordância de $75 \%$ entre os juízes, o valor foi obtido em um estilo diferente (pragmático) do qual havia sido inicialmente desenvolvido (reflexivo). A hipótese levantada envolve o fato de que possivelmente a situação de uso de tecnologia relatada no item chamou mais a atenção dos juízes do que a ação do indivíduo frente à situação apresentada. Tal item, assim como aqueles que não alcançaram valores satisfatórios (itens 2, 3 e 8), foram reescritos e passaram por nova avaliação de juízes, relatada posteriormente. 
Tabela 1.

Índice de Concordância entre juízes para os itens referentes aos estilos Ativo e Reflexivo.

\begin{tabular}{ccccccc}
\hline Estilo & Item & J1 & J2 & J3 & J4 & \% de concordância \\
\hline & 1 & P & A & A & A & 75,0 \\
Ativo & 2 & A & A & A & A & 100,0 \\
& 3 & T & A & A & A & 75,0 \\
& 4 & T & A & A & A & 75,0 \\
& 5 & A & A & A & A & 100,0 \\
& 6 & R & A & A & A & 75,0 \\
& 7 & A & A & A & P & 75,0 \\
& 8 & T & A & A & A & 75,0 \\
& 9 & T & A & A & A & 75,0 \\
& 10 & A & A & A & A & 100,0 \\
\hline Reflexivo & 1 & R & R & R & R & 100,0 \\
& 2 & T & R & R & T & 50,0 \\
& 3 & T & R & R & T & 50,0 \\
& 4 & R & R & A & R & 75,0 \\
& 5 & R & A & R & R & 75,0 \\
6 & P & P & P & R & 75,0 \\
& 7 & P & R & R & R & 75,0 \\
8 & A & A & R & R & 50,0 \\
& 10 & R & T & R & R & 75,0 \\
& R & R & R & R & 100,0 \\
\hline
\end{tabular}

Legenda: $\mathrm{A}=$ ativo, $\mathrm{R}=$ reflexivo, $\mathrm{T}=$ teórico, $\mathrm{P}=$ pragmático.

A seguir, os itens que compõem o estilo teórico foram avaliados. Indivíduos com esse estilo preferem aprender por meio de modelos, teorias e conceitos, bem como através de análises lógicas de ideias. Os dados da Tabela 2 indicam que, oito dos 10 itens obtiveram índices de concordância de $100 \%$ e dois itens apresentaram índices de concordância de 75\%. Novamente um dos itens apresentou concordância satisfatória, mas em estilo diferente do originalmente elaborado (item 3). Desenvolvido para ser um item do estilo teórico, o mesmo foi julgado pela maioria dos juízes como pertencente ao estilo ativo. Diante desse resultado inesperado, os autores optaram por sua reformulação e novo envio para avaliação dos juízes. De modo similar, a situação de uso de tecnologia relatada chamou mais a atenção dos juízes do que a ação do indivíduo propriamente dita. 
Tabela 2.

Índice de Concordância entre juízes para os itens referentes aos estilos Teórico e Pragmático.

\begin{tabular}{|c|c|c|c|c|c|c|}
\hline Estilo & Item & $\mathrm{J} 1$ & 32 & 33 & $\mathrm{J4}$ & \% de concordância \\
\hline & 1 & $\mathrm{~T}$ & $\mathrm{~T}$ & $\mathrm{~T}$ & $\mathrm{~T}$ & 100,0 \\
\hline & 2 & $\mathrm{~T}$ & $\mathrm{~T}$ & $\mathrm{~T}$ & $\mathrm{~T}$ & 100,0 \\
\hline & 3 & $A$ & $A$ & $A$ & $\mathrm{~T}$ & 75,0 \\
\hline \multirow[t]{11}{*}{ Teórico } & 4 & $\mathrm{~T}$ & $\mathrm{~T}$ & $\mathrm{~T}$ & $\mathrm{~T}$ & 100,0 \\
\hline & 5 & $\mathrm{~T}$ & $\mathrm{~T}$ & $\mathrm{~T}$ & $\mathrm{~T}$ & 100,0 \\
\hline & 6 & $\mathrm{~T}$ & $\mathrm{~T}$ & $\mathrm{~T}$ & $\mathrm{~T}$ & 100,0 \\
\hline & 7 & $\mathrm{~T}$ & $\mathrm{~T}$ & $\mathrm{R}$ & $\mathrm{T}$ & 75,0 \\
\hline & 8 & $\mathrm{~T}$ & $\mathrm{~T}$ & $\mathrm{~T}$ & $\mathrm{~T}$ & 100,0 \\
\hline & 9 & $\mathrm{~T}$ & $\mathrm{~T}$ & $\mathrm{~T}$ & $\mathrm{~T}$ & 100,0 \\
\hline & 10 & $\mathrm{~T}$ & $\mathrm{~T}$ & $\mathrm{~T}$ & $\mathrm{~T}$ & 100,0 \\
\hline & 1 & $P$ & $P$ & $\mathrm{P}$ & $P$ & 100,0 \\
\hline & 2 & $A$ & $A$ & $P$ & $\mathrm{P}$ & 50,0 \\
\hline & 3 & $P$ & $P$ & $P$ & $P$ & 100,0 \\
\hline & 4 & $P$ & $P$ & $P$ & $P$ & 100,0 \\
\hline \multirow[t]{6}{*}{ Pragmático } & 5 & $P$ & $P$ & $P$ & $P$ & 100,0 \\
\hline & 6 & $P$ & $P$ & $\mathrm{P}$ & $P$ & 100,0 \\
\hline & 7 & $P$ & $P$ & $\mathrm{P}$ & $\mathrm{P}$ & 100,0 \\
\hline & 8 & $\mathrm{P}$ & $P$ & $P$ & $\mathrm{~T}$ & 75,0 \\
\hline & 9 & $P$ & $P$ & $P$ & $P$ & 100,0 \\
\hline & 10 & $P$ & $P$ & $P$ & $\mathrm{P}$ & 100,0 \\
\hline
\end{tabular}

Legenda: $\mathrm{A}=$ ativo, $\mathrm{R}=$ reflexivo, $\mathrm{T}=$ teórico, $\mathrm{P}=$ pragmático.

Na mesma Tabela podem ser visualizados os dados do último estilo de aprendizagem avaliado, o pragmático. Indivíduos com estilo pragmático preferem as aplicações práticas de ideias, com o estabelecimento de uma relação clara entre os assuntos abordados e situações reais. Para esse estilo, oito itens obtiveram índices de concordância de 100\%, um item obteve índice de concordância de $75 \%$ e um item obteve índice de concordância de 50\%, sendo reescrito.

A reformulação dos itens que não apresentaram valores satisfatórios (quatro itens do estilo reflexivo, um item do estilo teórico e um item do estilo pragmático) ocorreu em mais duas etapas. Na primeira, os seis itens com índices de concordância insatisfatórios tiveram sua redação alterada na busca de deixar a compreensão dos itens mais clara. Assim, nessa segunda rodada de avaliação, três itens obtiveram índices de concordância de $100 \%$ e um item obteve índice de concordância de 75\%, sendo considerados satisfatórios. Dois itens, no entanto, permaneceram com índices de concordância insatisfatórios, de $50 \%$ e 
$25 \%$, referentes aos estilos teórico e reflexivo, respectivamente. Na segunda etapa de reformulação, os dois itens considerados insatisfatórios foram substituídos. Com a substituição, os índices de concordância alcançados foram de $100 \%$ e $75 \%$. Dessa forma, todos os itens da escala atingiram índices de concordância acima de $75 \%$, sendo considerados satisfatórios.

Uma segunda análise refere-se aos coeficientes Kappa, cujos dados são apresentados na Tabela 3. Para tanto, a classificação de cada juiz foi comparada com a classificação de um juiz "ideal", representada pelas respostas esperadas para cada item, ou seja, sua classificação no estilo para o qual foi, originalmente, desenvolvido.

Tabela 3.

Estatística Kappa e valores de p (entre parênteses) para a avaliação dos juízes por estilo de aprendizagem.

\begin{tabular}{|c|c|c|c|c|c|c|}
\hline Juiz & Estatística & Critério & A & $\mathrm{R}$ & $\mathrm{T}$ & $\mathrm{P}$ \\
\hline \multirow{3}{*}{$\begin{array}{c}\text { Juiz } \\
1\end{array}$} & \multirow{3}{*}{$\begin{array}{c}0,767 \\
(0,0001)\end{array}$} & $\begin{array}{l}\text { Número de itens classificados } \\
\text { em cada área }\end{array}$ & 4 & 10 & 14 & 12 \\
\hline & & Acertos & 4 & 9 & 10 & 10 \\
\hline & & Porcentagem de acerto & 40 & 90 & 100 & 100 \\
\hline \multirow{3}{*}{$\begin{array}{c}\text { Juiz } \\
2\end{array}$} & \multirow{3}{*}{$\begin{array}{c}0,867 \\
(0,0001)\end{array}$} & $\begin{array}{c}\text { Número de itens classificados } \\
\text { em cada área }\end{array}$ & 11 & 6 & 11 & 12 \\
\hline & & Acertos & 10 & 6 & 10 & 10 \\
\hline & & Porcentagem de acerto & 100 & 60 & 100 & 100 \\
\hline \multirow{3}{*}{$\begin{array}{c}\text { Juiz } \\
3\end{array}$} & \multirow{3}{*}{$\begin{array}{c}0,933 \\
(0,0001)\end{array}$} & $\begin{array}{c}\text { Número de itens classificados } \\
\text { em cada área }\end{array}$ & 11 & 10 & 9 & 10 \\
\hline & & Acertos & 10 & 9 & 9 & 10 \\
\hline & & Porcentagem de acerto & 100 & 90 & 90 & 100 \\
\hline \multirow{3}{*}{$\begin{array}{c}\text { Juiz } \\
4\end{array}$} & \multirow{3}{*}{$\begin{array}{c}0,933 \\
(0,0001)\end{array}$} & $\begin{array}{c}\text { Número de itens classificados } \\
\text { em cada área }\end{array}$ & 9 & 10 & 11 & 10 \\
\hline & & Acertos & 9 & 10 & 10 & 9 \\
\hline & & Porcentagem de acerto & 90 & 100 & 100 & 90 \\
\hline Total & & & 35 & 36 & 45 & 44 \\
\hline
\end{tabular}

Nota. $\mathrm{A}=$ ativo; $\mathrm{R}=$ reflexivo; $\mathrm{T}=$ teórico; $\mathrm{P}=$ pragmático.

Tomando-se os valores estabelecidos na literatura científica (Fleiss, 1981), pode-se verificar que todos os juízes apresentaram uma concordância excelente, ou seja, acima de 0,75. De um modo geral, observa-se que houve uma tendência dos juízes em classificar menos itens nos estilos de aprendizagem ativo $(n=35)$ e reflexivo $(n=36)$ e mais itens nos estilos teórico $(n=45)$ e pragmático $(n=44)$. Convém destacar que dois juízes apresentaram menores porcentagens de acertos que os demais. O juiz 1 acertou apenas $40 \%$ dos itens 
no estilo ativo, fato este já destacado na análise do índice de concordância, bem como o juiz 2 no estilo reflexivo (60\%).

\section{DISCUSSÃO}

A pesquisa aqui relatada teve, como objetivo, a busca pelas evidências de validade de conteúdo de uma escala em processo de desenvolvimento, a Escala de Estilos de Aprendizagem em Situações de Uso de Tecnologias. O estudo desenvolvido buscou investigar a clareza, representatividade e relevância dos itens por meio da análise de juízes. Para isso, dois métodos foram utilizados: a porcentagem de concordância e a estimativa do coeficiente Kappa.

De modo geral, os resultados apontaram a adequação da escala às áreas que ela pretende avaliar. A primeira etapa de avaliação de juízes se mostrou bastante positiva, com a maioria dos itens sendo classificada de modo adequado pelos juízes e alcançando índices de concordância considerados adequados na primeira rodada de avaliação ( $85 \%$ dos itens), segundo os critérios adotados a partir dos apontamentos de Pasquali (2010). Duas novas rodadas de avaliação foram conduzidas. A primeira teve como foco uma nova redação dos itens e a segunda voltou-se para substituição dos itens que continuaram apresentando problemas, segundo a avaliação dos juízes. Como resultado, seis itens foram reformulados, os quais não haviam alcançado o IC almejado (sendo 4 do estilo reflexivo, 1 do estilo teórico e 1 do estilo pragmático), possibilitando, assim, que todos os itens alcançassem valores satisfatórios para sua manutenção no instrumento.

Chama a atenção o fato de que o estilo ativo foi o único em que os juízes apresentaram concordância em todos os itens já na primeira avaliação. Por outro lado, os itens do estilo reflexivo foram os que apresentaram maiores problemas, visto que três deles não alcançaram o valor mínimo de concordância estabelecido como satisfatório, bem como outro item alcançou esse valor, mas em estilo diferente daquele para o qual originalmente foi desenvolvido.

Os resultados do segundo método utilizado, do coeficiente Kappa, o qual teve como objetivo a verificação das classificações feitas pelos juízes e os quatro estilos avaliados, também apontou para resultados satisfatórios, conforme valores apontados por Fleiss (1981), não havendo nenhum juiz que tenha se mostrado inadequado. De modo semelhante, não se fez notar a predominância de maiores índices de acertos em um estilo específico. Tal dado permite inferir que, de modo geral, as definições fornecidas para os juízes mostraram-se adequadas e suficientes para a tarefa solicitada.

Os resultados desse estudo inicial com a Escala de Estilos de Aprendizagem em Situações de Uso de Tecnologias, apresentado neste artigo, demonstram a adequação dos itens da escala ao modelo teórico que foi utilizado como base para sua construção (Alonso et al., 1997). Após terem sido 
encontradas evidências positivas de validade de construto para a escala, sua primeira versão está pronta e adequada para ser testada, de forma empírica, em outros estudos de investigação de suas qualidades psicométricas junto a outras amostras da população brasileira, até que 0 instrumento possa ser disponibilizado, de forma segura, para uso profissional.

Sua relevância ampara-se no fato de que, faz-se notar, mais atualmente, o reconhecimento da necessidade de obtenção de uma compreensão mais profunda dos estudantes, suas diferenças em termos de aprendizagem, dificuldades, estilos de aprendizagem e predisposição para o uso de certos tipos de tarefas. Nesse sentido, a literatura tem destacado que o respeito aos diferentes estilos dos estudantes, por meio da adoção de diferentes abordagens de aprendizagem (percepção, sensorial, intuitiva, processamento, reflexiva, ativa, visual, auditiva, sequencial, global), pode favorecer a sua aprendizagem (Ozdemir et al., 2016), de forma a beneficiar, inclusive alunos que apresentam algum tipo de deficiência (Ibrahim, 2016).

Além disso, a importância de uma escala de estilos de aprendizagem voltada para situações de uso de tecnologias deve-se à própria realidade da atual sociedade da informação ou do conhecimento. Sabe-se que as influências sociais, politicas e econômicas que têm sido geradas pelos avanços tecnológicos têm levado a uma modificação importante dos paradigmas que regem os sistemas educacionais convencionais, de modo a favorecerem o surgimento de novos modelos de ensino e aprendizagem baseados em dimensões multiculturais e caracterizados pelo desaparecimento das barreiras como espaço e tempo (Acosta-Castillo, 2016), por meio de diferentes plataformas tecnológicas virtuais. A incorporação dessas ferramentas, ainda de acordo com a autora, deve ser valorizada e incentivada visto que permite a potencialização das habilidades e estratégias relacionadas às competências comunicativas, cognitivas e informáticas que facilitam a interação pessoal, a difusão da informação e a queda de barreiras que limitam a aquisição de conhecimento.

Isso porque, evidências ressaltadas na literatura científica têm apontado que a incompatibilidade entre os estilos de aprendizagem dos alunos e as estratégias empregadas pelo professor podem exercer um impacto negativo em sala de aula (Rezaeinejad, Azizifar, \& Gowbary, 2015). Estes mesmos autores citam, como alguns dos possíveis motivos, o fato de que muitos dos professores são produtos de um sistema educacional tradicional, não estão cientes dos estilos de seus alunos e tendem a utilizar um número limitado de estilos de ensino, dentro de uma zona de conforto marcada pelos conhecimentos que já possuem. 


\section{CONSIDERAÇÕES FINAIS}

Diante do exposto ao longo do texto, a relevância do desenvolvimento de um instrumento de avaliação de estilos de aprendizagem em situações de uso das tecnologias da informação e comunicação tem sido, cada vez mais, enfatizada perante a escassez de instrumental para tal propósito, especialmente no Brasil, e às novas particularidades da atual sociedade, fortemente apoiada nas novas tecnologias. Ainda que, nos últimos anos, os estudos realizados com o objetivo de personalizar os ambientes de aprendizagem online, de acordo com as necessidades individuais dos alunos e de determinar seus comportamentos nesses ambientes venham adquirindo uma importância considerável (Ozdemir et al., 2016), essa temática permanece praticamente inexplorada no país. Neste sentido, a Escala de Estilos de Aprendizagem em Situações de Uso de Tecnologias busca suprir uma lacuna no campo da avaliação psicológica, contribuindo para compreensão das preferências dos indivíduos ao aprender em uma realidade transformada pelos recursos tecnológicos.

Uma reflexão acerca dos procedimentos metodológicos adotados no estudo indica, como possível limitação, o número dos juízes (menor do que o desejável), assim como suas qualificações, considerando-se que, ainda que trabalhem na área da avaliação psicológica (e tenham sido priorizados em relação a sua qualificação), não estudam diretamente a temática dos estilos de aprendizagem. Tais características podem ter exercido alguma influência nos resultados. Do mesmo modo, ainda que as definições de cada estilo adotadas pelos pesquisadores tenham seguido fielmente o modelo teórico que embasou 0 instrumento, uma hipótese que se levanta envolve a possibilidade de que a taxonomia selecionada possa não ter sido clara o suficiente para embasar alguns julgamentos. Tal situação pode ter ocorrido especificamente em relação a alguns itens que apresentaram resultados diferentes do esperado (envolvendo a classificação de itens em estilos diferentes daqueles para os quais foi hipotetizado ou porcentagem de concordância entre os juízes menor do que a esperada).

Embora os resultados tenham se mostrado positivos, é importante salientar que eles são decorrentes de um estudo inicial. Neste sentido, o presente estudo limitou-se à análise de um tipo específico de evidências de validade. Outros estudos são necessários até que o instrumental possa ser disponibilizado para uso profissional.

Almeja-se que o instrumento, ao permitir o conhecimento dos estilos preferenciais de aprender dos estudantes em contexto de uso de tecnologias, possibilite um maior aproveitamento de aprendizagem, à medida em que a apresentação do conteúdo a ser estudado esteja mais direcionado ao estilo do aluno, tal como enfatizado por Resende e Dorça (2015), de modo que tais informações possam ser utilizadas pelos professores, futuramente e em um 
cenário ideal, na constituição de um processo de avaliação personalizada e de adaptação do conteúdo às características dos estudantes.

\section{DECLARAÇÃO DE CONFLITOS DE INTERESSE}

Os autores declaram não haver conflitos de interesse.

\section{REFERÊNCIAS}

Acosta-Castillo, L. (2016). La relación entre los estilos de aprendizaje y el uso de las tecnologías de información y comunicación en educación de personas adultas. Revista Eletronica Educare, 20(3), 1-18.

Alexandre, N. M. C., \& Coluci, M. Z. O. (2011). Validade de conteúdo nos processos de construção e adaptação de instrumentos de medidas. Ciência e Saúde Coletiva, 16(7), 3061-3068.

Alonso, C. M., Gallego, D. J., \& Honey, P. (1997). Los estilos de aprendizaje: Procedimientos de diagnostico y mejora (7a. ed.). Bilbao: Ediciones Mensajero.

Alves, P., Miranda, L., \& Morais, C. (2014). Estilos de aprendizagem e utilização de recursos digitais no ambiente colaborativo Sakai. In VI Congreso Mundial de Estilos de Aprendizaje, Pontificia Universidad Católica Del Perú, Perú.

Butzke, M.A., \& Alberton, A. (2017). Estilos de aprendizagem e jogos de empresa: a percepção discente sobre estratégia de ensino e ambiente de aprendizagem. REGE - Revista de Gestão, 24(1), 72-84. doi: $10.1016 /$ j.rege.2016.10.003

Cué, J. L. G., Rincón, J. A. S., \& García, C. M. A. (2009). Instrumentos de medición de estilos de aprendizaje. Journal of Learning Styles, 2(4), 3-21.

Castells, M. (2010). The rise of the network society: The information age: Economy, society, and culture (Vol. 1). Oxford: Wiley-Blackwell.

Coll, C., \& Monereo, C. (2010). Educação e aprendizagem no século XXI: Novas ferramentas, novos cenários, novas finalidades. In C. Coll \& C. Monereo (Orgs.), Psicologia da educação virtual: Aprender e ensinar com as tecnologias da informação e da comunicação (pp. 15-46). Porto Alegre, RS: Artmed.

Cuevas, J. (2015). Is learning styles-based instruction effective? A comprehensive analysis of recent research on learning styles. Theory and Research in Education, 13(3), 1-26.

Dunn, R. S., \& Dunn, K. J. (1978). Teaching students through their individual learning styles: A practical approach. Reston: Prentice Hall.

Felder, R. M., \& Silverman, L. K. (1988). Learning and teaching styles in engineering education. Engineering Education, 78(7), 674-681. 
Fleiss, J. L. (1981). The measurement of inter-rather agreement. In J. L. Fleiss (Org.), Statistical methods for rates and proportions (pp. 212-236). New York, NY: John Wiley \& Sons Inc.

Fonseca, R., Silva, P., \& Silva, R. (2007). Acordo inter-juízes: O caso do coeficiente kappa. Laboratório de Psicologia, 5(1), 81-90.

Gardner, H. (1983). Frames of Mind. New York, NY: Basic Book Inc.

Gocus, A., \& Ertek, G. (2016). Learning and personal attributes of university students in predicting and classifying the learning styles: Kolb's nine-region versus four-region learning styles. Procedia - Social and Behavioral Sciences, 217, 779-789.

Gregorc, A.R. (1982). Style Delineator. Maynard: Gabriel Systems.

Gungor, F., Sofraci, G., Çelik, D., \& Yayli, D. (2016). Learning styles of English preparatory school students and the relationship of their proficiency with learning styles and gender. Kastamonu Education Journal, 24(3), 707-716.

Ibrahim, Z. (2016). Needs analysis for graphic design learning module based on technology \& learning styles of deaf students. Cogent Education, 3, 1-14.

Kolb, D. A. (1984). Experiential learning: Experience as the source of learning and development. New Jersey: Prentice-Hall.

Landis, J. R., \& Koch, G. G. (1977). The measurement of observer agreement for categorical data. Biometrics, 33, 159-74.

Li, Y., Medwell, J., Wray, L., \& Liu, X. (2016). Learning styles: A review of validity and usefulness. Journal of Education and Training Studies, 4(10), 90-94.

Mayaute, L.M.E. (2011). Análisis psicométrico del Cuestionário de Honey y Alonso de Estilos de Aprendizaje (CHAEA) con los modelos de la Teoría Clásica de los tests y Rasch. Persona, 14, 71-109.

Ozdemir, A., Alaybeyoglu, A., Mulayin, N., \& Balbal, K.F. (2016). Performance evaluation of learning styles based on fuzzy logic inference system. Computer Applications in Engineering Education, 24(6), 853-865.

Okada, A., Barros, D. M. V., \& Santos, L. (2008). Discutindo estilos de aprendizagem com tecnologias do projeto OpenLearn para videoconferência e mapeamento do conhecimento. Revista Estilos de Aprendizagem,1(2), 110-129.

Oliveira, K. L., Inácio, A. L. M., \& Buriolla, H. L. (2016). Diferenças considerando ano escolar no ensino fundamental: Um estudo com estilos intelectuais. Argumentos Pró-Educação, 1(3), 408-422.

Oliveira, K.L., Santos, A.A.A., \& Scacchetti, F.A.P. (2016). Medidas de estilos de aprendizagem para o ensino fundamental. Psicologia Escolar e Educacional, 20(1), 127-136.

Pasquali, L. (1999). Testes referentes a constructo: Teoria e modelo de construção. In L. Pasquali (Org.), Instrumentos psicológicos: Manual prático de elaboração (pp.37-71). Brasília: LabPAM/IBAP. 
Portilho, E. M. L. (2004). Aprendizaje universitario: Un enfoque metacognitivo. Tese de Doutorado. Universidad Complutense de Madrid.

Rajper, S., Shaikh, N.A., Shaikh, Z.A., \& Mallah, G.A. (2016). Automatic detection of learning styles on learning management systems using data mining technique. Indian Journal of Science and Technology, 9(15), 1-5.

Rezaeinejad, M., Azizifar, A., \& Gowbary, H. (2015). The study of learning styles and its relationship with educational achievement among Iranian high school students. Procedia: Social and Behavioral Sciences, 11, 218-224.

Resende, D.T., \& Dorça, F.A. (2015). Recomendação de conteúdo personalizada com base em estilos de aprendizagem: Uma abordagem prática. Revista Brasileira de Informática na Educação, 23(3), 11-25.

Sternberg, R. J. (1988). Mental self-government: A theory of intellectual styles and their development. Human Development, 31(4), 197-224.

Sternberg, R. J. (1994). Allowing for Thinking Styles. Educational Leadership, $52(3), 36-40$.

\section{Sobre os autores}

Rodrigo Hipólito Roza é bacharel em Ciência da Computação pela Universidade Paulista, mestre em Ciência da Informação e doutor em Psicologia pela Pontifícia Universidade Católica de Campinas. É docente do Centro de Economia e Administração da Pontifícia Universidade Católica de Campinas.

rodrigo.roza@gmail.com

Solange Muglia Wechsler é psicóloga pela Pontifícia Universidade Católica do Rio de Janeiro, mestre e doutora em Psicologia pela University of Georgia, e possui pós-doutorado pela University of Georgia e pela University of Buffalo. É docente pesquisadora do curso de Pós-Graduação stricto sensu em Psicologia pela Pontifícia Universidade Católica de Campinas. Bolsista Produtividade em Pesquisa CNPq. wechsler@lexxa.com.br

Tatiana de Cassia Nakano é psicóloga, mestre e doutora em Psicologia pela Pontifícia Universidade Católica de Campinas, e possui pós-doutorado pela Universidade São Francisco. É docente pesquisadora do curso de Pós-Graduação stricto sensu em Psicologia pela Pontifícia Universidade Católica de Campinas. Bolsista Produtividade em Pesquisa CNPq. tatiananakano@hotmail.com

Certificamos que todos os autores participaram suficientemente do trabalho para tornar pública sua responsabilidade pelo conteúdo. A contribuição de cada autor pode ser atribuída como se segue: Rodrigo Hipólito Roza contribuiu com a execução de todas as etapas do estudo; Solange Muglia Wechsler orientou e revisou o trabalho. Tatiana de Cassia Nakano contribuiu com as análises e a elaboração do texto final. 
$1^{a}$ revisão em: 16/03/2017

2a revisão em: 07/04/2017

Aceito em: 25/05/2017 